\title{
MULTI: a program for multicomponent analysis of spectra
}

\author{
Marc Eberhard, Bernd Leister' and Cornelia Stoppkotte'
}

\section{Introduction}

The constituents of solutions may be quantified spectrophotometrically by multicomponent analysis provided that (i) the composition of the solution to be analyzed is known, (ii) the constituents to be quantitated have distinct spectral properties and (iii) the spectrum of the solution to be analyzed is a linear superposition of the spectra of the pure constituents. We have implemented MULTI, a program to decompose the spectrum of a solution into contributions of constituents by multicomponent analysis. The input of MULTI consists of ASCII files containing the standard spectra, i.e. the spectra of the pure components, and that of the solution to be analyzed. ASCII files generated by the Kontron Uvikon 930 and the Hewlett-Packard 8452A spectrophotometer can be read without preprocessing. MULTI allows the user to average spectra in order both to increase the signal-to-noise ratio and obtain estimates about the quality of the data, i.e. standard deviations. A linear least-squares fit is used for the analysis (Press et al., 1989). Experimental data are weighted by the inverse of the squared standard deviation. As a result MULTI provides the concentration and its standard deviation of each component. Up to eight components may be included in the analysis. Each spectrum may contain up to 500 individual data points.

As an example we have quantitated the aromatic residues phenylalanine, tyrosine and tryptophan, of three proteins of known sequence using second-derivative spectroscopy according to the method of Levine and Federici (1982). The test proteins were aspartate aminotransferase from Escherichia coli (AAT), an Ala to Trp mutant of AAT (AAT-A403W, C.Stoppkotte, unpublished) and an engineering domain of AAT (P-domain, Herold et al., 1991). $N$-Acetyl-phenylalanine ethyl ester, $N$ acetyl-tyrosine ethyl ester and mellitin were used as model compounds for phenylalanine, tyrosine and tryptophan respectively. Each of the standards and the proteins to be analyzed were dissolved in $10 \mathrm{mM} \mathrm{Na(2-(4-(2-hydroxyl)-1-}$ piperazinyl)-ethanesulfonate) $\mathrm{pH} 7.4,1 \mathrm{mM}$ dithioerythritol, $1 \mathrm{mM}$ ethylenedinitrilotetraacetic acid, $6 \mathrm{M}$ guanidine hydrochloride, except $N$-acetyl-tyrosine ethyl ester which was dissolved in 55\% methanol (Levine and Federici, 1982). The solutions of the standard compounds were quantitated using the

'Biozentrum, Universin' Basel, Department of Biophysical Chemistn', Klingelhergstrasse 70, $\mathrm{CH}-4056$ Basel. Sivizerland extinction coefficients given by Levine and Federici (1982). A Hewlett-Packard 8452A diode-array spectrophotometer with $1 \mathrm{~nm}$ resolution was used to obtain the spectra and their second derivatives with respect to the wavelength; MULTI was used to perform the multicomponent analysis. The concentration of phenylalanine of the test proteins was obtained by analysis of the spectra from 245 to $265 \mathrm{~nm}$, that of tyrosine and tryptophan from spectra between 280 and $300 \mathrm{~nm}$. The concentrations of phenylalanine, tyrosine and tryptophan determined in this way were divided by the number of the respective residues expected from the amino acid sequence of the protein in order to obtain the concentration of the protein (Table I).

MULTI can be run on any PC-or AT-compatible computer equipped with at least one floppy drive, Hercules, CGA, EGA or VGA graphics and 512 kbytes of RAM. A numerical coprocessor is supported if present. MS-DOS or PC-DOS v. 2.0 or higher is required. The program MULTI, some utilities and a comprehensive manual of MULTI are available on $51 / 4$-in. or $31 / 2$-in. diskettes from (M.Eberhard).

Table I. Examples of protein quantitation using MULTI

\begin{tabular}{|c|c|c|c|c|c|c|c|c|c|}
\hline Protein & {$[\text { Phe }]^{2}$} & [Tyr] & [Trp] & $n_{\mathrm{Phe}}{ }^{\mathrm{b}}$ & $n_{\mathrm{Tyr}}$ & $n_{\text {Trp }}$ & [Phe] & [Tyr] & [Trp] \\
\hline & $(\mu \mathrm{M})$ & $(\mu \mathrm{M})$ & $(\mu \mathrm{M})$ & & & & $\begin{array}{l}n_{\text {Ptce }} \\
(\mu \mathrm{M})\end{array}$ & $\begin{array}{l}n_{T y r} \\
(\mu M)\end{array}$ & $\begin{array}{l}n_{\text {Typ }} \\
(\mu \mathrm{M})\end{array}$ \\
\hline AAT & 54.0 & 26.1 & 12.4 & 20 & 11 & 5 & 2.70 & 2.37 & 2.48 \\
\hline AAT & 76.4 & 39.9 & 22.3 & 20 & 11 & 6 & 3.82 & 3.63 & 3.71 \\
\hline P-domain & 77.1 & 62.7 & 34.6 & 12 & 9 & 5 & 6.42 & 6.97 & 6.92 \\
\hline
\end{tabular}

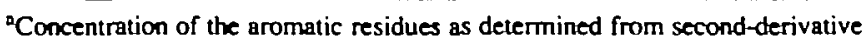
spectra using MULTI.

${ }^{b}$ Number of the aromatic residues obtained from the amino acid sequence of the proteins.

\section{References}

Herold,M., Leistler.B., Hage,A., Luger,K, and Kirschner,K. (1991) Autonomous folding and coenzyme binding of the excised pyridoxal 5'-phosphate binding domain of aspartate aminotransferase from Escherictio coli. Biochemistry, 30, 3612-3620.

Levine,L.L. and Federici,M.M. (1982) Quantitation of aromatic residues in proctins: model compounds for second-derivative spectroscopy. Biochemistry, 21, 2600-2606.

Press,W.H., Flannery,B.P., Teukolsky,S.A. and Vetterling,W.T. (1989) Numerical Recipes in Pascal. Cambridge University Press, Cambridge. Chapters 2.9 and 14.3 .

Circle No. 16 on Reader Enquiry Card 